\title{
INTRODUÇÃO ÀS TÉCNICAS DE REFLEXÃO ESPECULAR E REFLEXÃO-ABSORÇÃO NO INFRAVERMELHO: (1) REFLEX ̃̃O ESPECULAR
}

\author{
Benedito Cláudio Trasferetti e Celso Ulysses Davanzo
}

Instituto de Química, Universidade Estadual de Campinas, CP 6154, 13083-970 Campinas - SP

Recebido em 13/10/99; aceito em 26/6/00

\begin{abstract}
INTRODUCTION TO THE SPECULAR REFLECTION AND REFLECTION-ABSORPTION TECHNIQUES IN THE INFRARED: (1) SPECULAR REFLECTION: This paper is the first part of an article aimed to present theoretical basis as well as some applications of two infrared reflection techniques: specular reflection and reflection-absorption. It is emphasyzed how the Kramers-Krönig analysis of reflection data can be useful in both retrieving optical constants and making spectral analysis possible. Examples of vitreous, powdered and liquid samples are given.
\end{abstract}

Keywords: infrared; specular reflection; Kramers-Krönig analysis.

\section{INTRODUÇÃO}

A espectroscopia vibracional tornou-se uma técnica bastante acessível depois da ampla difusão de espectrômetros de infravermelho com transformada de Fourier. O fato de ser possível a obtenção de espectros digitalizados com alta razão sinal/ruído tornou a técnica potencialmente importante para novas aplicações.

Nesse contexto, os pesquisadores, acostumados com a simplificação tradicional da interpretação espectral proporcionada pelas técnicas de transmissão, passaram a olhar com mais atenção às possibilidades das técnicas que fazem uso do modo reflectância, seja ela atenuada ${ }^{1,2}$, difusa ${ }^{3,4}$ ou especular ${ }^{5,6}$. Essas técnicas são de grande interesse na análise não destrutiva e pela possibilidade de obtenção de espectros in situ e de interpretações a partir da dependência do espectro com o índice de refração complexo do material

Nessas técnicas, é de fundamental importância o uso da teoria da Óptica ${ }^{7-10}$, que embora tenha sido desenvolvida já há algum tempo, nunca teve sua aplicação tão acessível e barata quanto agora devido ao atual estágio de desenvolvimento da tecnologia computacional e eletrônica. Com isso, a aplicação da teoria da Óptica à pesquisa de rotina e a revisão das técnicas espectroscópicas de reflexão são muito oportunas.

Nesses dois artigos, portanto, retomamos duas técnicas de reflexão especular no infravermelho com o objetivo de realçar as potencialidades de cada uma, o que é interessante num contexto de ampla difusão das técnicas de transmitância. O presente artigo trata da técnica de reflexão especular. Enfatizaremos a sua utilização em casos onde as técnicas convencionais de transmissão apresentam inconvenientes na amostragem, como é o caso de materiais vítreos e cerâmicos. Enfatizaremos, também, a obtenção de constantes ópticas, que são os parâmetros que viabilizam simulações espectrais, a partir desse tipo de técnica. A segunda parte do artigo tratará da técnica de reflexão-absorção, onde serão enfocados os desdobramentos de óptica longitudinal (LO) e transversal (TO) e sua aplicação à caracterização de filmes finos depositados em diversos substratos.

Em ambos os casos, estaremos enfatizando o ferramental teórico e computacional, sem o qual, na maioria dos casos, é impossível o entendimento dos resultados experimentais. Em uma futura série de artigos, mostraremos como todas as técnicas de reflectância no infravermelho podem ser aplicadas, experimentalmente e em diferentes situações.

\section{ASPECTOS TEÓRICOS}

A reflectância especular ocorre na grande maioria das superfícies que constituem a fronteira entre fases ${ }^{7-10}$ (Figura 1). A natureza da radiação refletida depende da natureza do feixe incidente, bem como das propriedades ópticas dos meios em questão. Assim, podem-se utilizar dados de reflectância para se determinar as propriedades ópticas das substâncias de interesse do espectroscopista, se as características do processo de reflexão são bem entendidas. Contudo, as constantes ópticas não podem ser identificadas a partir das características estruturais das curvas de reflectância especular. Para tal fim, conta-se com análises matemáticas complexas para a obtenção de dados tanto qualitativos quanto quantitativos.

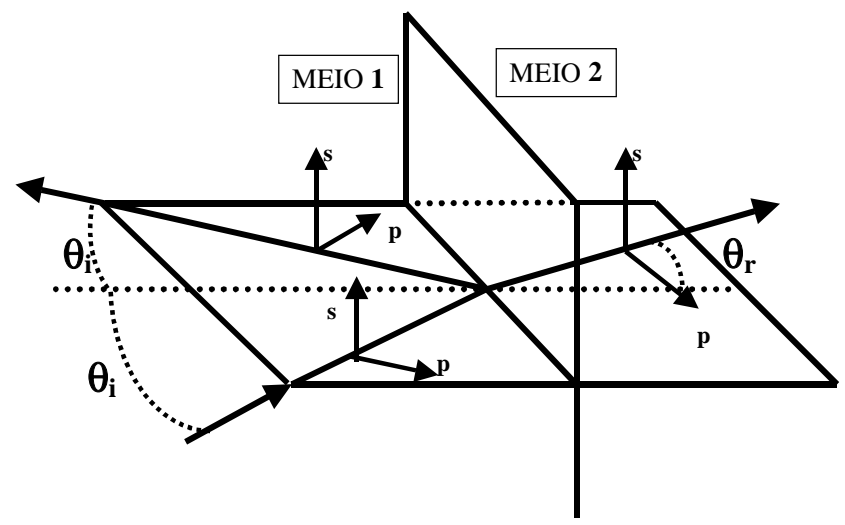

Figura 1. Os efeitos da descontinuidade do meio de propagação na radiação eletromagnética.

Os índices de refração $\mathrm{n}$ e de absorção $\mathrm{k}$ de um material, que juntos são chamados de constantes ópticas, são os parâmetros que determinam a interação entre a luz e o material numa interface descontínua. Eles são as partes real e imaginária do índice de refração complexo $\tilde{n}=n+i k$.

$\mathrm{O}$ índice de absorção $\mathrm{k}(\operatorname{Im}(\tilde{\mathrm{n}})=\mathrm{k})$ relaciona-se à absortividade na base $10, \alpha$, pela expressão:

$\alpha=\frac{4 \pi \mathrm{k}}{2,303 \lambda_{0}}$

onde $\lambda_{0}$ é o comprimento de onda no vácuo. Convem ressaltar que a absortividade está relacionada com a Absorbância 
(- $\log ($ Transmitância)) pela expressão $A=\alpha l$, onde 1 é a espessura da amostra.

As constantes ópticas determinam a amplitude, a polarização e a fase da luz refletida e transmitida na interface de dois materiais diferentes através das equações de Fresnel. Teoricamente, essas equações requerem, para sua dedução, que a interface seja plana e que $\mathrm{n}$ e $\mathrm{k}$ variem abruptamente através da normal da interface ${ }^{8}$. Tal interface ideal, onde as condições de fronteira possam ser exatamente aplicadas às equações eletromagnéticas de Maxwell, é uma abstração que pode ser aproximada, mas nunca alcançada em experimentos. Tal consideração, entretanto, será feita no presente trabalho.

Na Figura 1 são apresentados os parâmetros envolvidos nas equações de Fresnel. Estas são escritas em função dos índices de refração dos dois meios ( $\tilde{n}_{1}$ e $\tilde{n}_{2}$ ) e dos ângulos de incidência $\left(\theta_{\mathrm{i}}\right)$ e de refração $\left(\theta_{\mathrm{t}}\right)^{8}$. Existem quatro relações de Fresnel para uma única interface: uma para cada polarização para as ondas refletida e transmitida. Há várias notações usadas para denotar a polarização da luz. De acordo com a notação elipsométrica de Muller, os subscritos p e s referem-se às polarizações paralela e perpendicular ao plano de incidência, respectivamente (Figura 1).

A reflectividade é definida como sendo a razão entre a amplitude do campo elétrico refletido e a do incidente para cada número de onda. Já a reflectância $\mathrm{R}$ é a razão entre os fluxos de energia nos feixes refletido e incidente e é a grandeza que se pode obter experimentalmente em função do número de onda. Essas duas grandezas guardam entre si a seguinte relação:

$$
\mathrm{R}=\tilde{\mathrm{r}} \tilde{\mathrm{r}}^{*}
$$

onde o sobrescrito $\sim$ representa uma grandeza complexa e $\tilde{\mathrm{r}}^{*} \mathrm{re}-$ presenta o complexo conjugado da reflectividade complexa.

As equações de Fresnel para a reflectividade são as seguintes:

$$
\tilde{\mathrm{r}}_{\mathrm{p}}=\frac{\tilde{\mathrm{n}}_{2} \cos \theta_{\mathrm{i}}-\tilde{\mathrm{n}}_{1} \cos \theta_{\mathrm{t}}}{\tilde{\mathrm{n}}_{2} \cos \theta_{\mathrm{i}}-\tilde{\mathrm{n}}_{1} \cos \theta_{\mathrm{t}}}
$$

$$
\tilde{\mathrm{r}}_{\mathrm{s}}=\frac{\tilde{\mathrm{n}}_{1} \cos \theta_{\mathrm{i}}-\tilde{\mathrm{n}}_{2} \cos \theta_{\mathrm{t}}}{\tilde{\mathrm{n}}_{1} \cos \theta_{\mathrm{i}}-\tilde{\mathrm{n}}_{2} \cos \theta_{\mathrm{t}}}
$$

onde os subscritos 1 e 2 se referem aos meios de incidência e de refração respectivamente.

A radiação eletromagnética sofre mudança de fase quando reflete numa interface; a reflectividade complexa em função dessa mudança de fase é dada por:

$$
\tilde{\mathrm{r}}=\sqrt{\mathrm{R}} \exp (\mathrm{i} \delta)
$$

onde $\delta$ é o ângulo de fase.

À incidência normal, a reflectância $R$, que nessa condição é independente da polarização, é dada pela seguinte equação, considerando-se que o meio de incidência seja o ar, cujo índice de refração pode ser considerado igual a 1 :

$$
R=\frac{(n-1)^{2}+k^{2}}{(n+1)^{2}+k^{2}}
$$

onde $\mathrm{n}$ e k são as constantes ópticas do material. Tal equação é independente da polarização da luz. Usando-se as equações 3 ou 4 e 5, podem-se deduzir equações separadas para os índices de refração e de absorção, num dado comprimento de onda $\lambda$ e à incidência normal $\left(\theta_{\mathrm{i}}=0^{0}\right)$ :

$$
\begin{aligned}
& \mathrm{n}=\frac{1-\mathrm{R}}{1-2 \sqrt{\mathrm{R}} \cos \delta+\mathrm{R}} \\
& \mathrm{k}=\frac{2 \sqrt{\mathrm{R}} \operatorname{sen} \delta}{1-2 \sqrt{\mathrm{R}} \cos \delta+\mathrm{R}}
\end{aligned}
$$

Estas equações são válidas para reflexão de superfícies planas em amostras de espessura semi-infinita. Uma amostra de espessura semi-infinita é aquela que permite a análise da reflectância especular apenas da sua primeira superfície, sem a interferência de reflexão ocorrendo na sub-superfície.

É importante ressaltar que tendo-se em mãos os índices $\mathrm{n}$ e $\mathrm{k}$, não somente o índice de refração complexo, mas também a constante dielétrica complexa $\left(\widetilde{\varepsilon}=\varepsilon_{1}+i \varepsilon_{2}\right)$ poderá ser obtida, através da fórmula de Maxwell ${ }^{10}$ :

$\tilde{\mathrm{n}}=\sqrt{\varepsilon \mu}$

sendo $\mu$ a permeabilidade magnética do material. Para o caso de substâncias não magnéticas $\mu=1$, fazendo com que a fórmula de Maxwell se reduza: $\tilde{\mathrm{n}}=\sqrt{\widetilde{\varepsilon}}$. Assim, têm-se as seguintes expressões para $\mathrm{e}_{1} \mathrm{e} \mathrm{e}_{2}{ }^{10}$ :

$$
\begin{aligned}
& \varepsilon_{1}=\mathrm{n}^{2}-\mathrm{k}^{2} \\
& \varepsilon_{2}=2 \mathrm{nk}
\end{aligned}
$$

Uma descrição mais detalhada das implicações da conexão entre índice de refração complexo e constante dielétrica complexa, bem como de outros parâmetros que podem ser calculados a partir dos mesmos, é dada por Bertie e seus colaboradores ${ }^{11}$.

\section{A análise de Kramers-Krönig}

Através da observação das equações 7 e 8 , pode-se ver com clareza que a obtenção das constantes ópticas n e k, através de medidas de reflectância $(\mathrm{R})$, só é possível com a recuperação do ângulo de fase $\delta$ - a parte imaginária do logaritmo da reflectividade complexa. A recuperação do ângulo de fase pode ser concretizada via uma expressão matemática chamada de relação de dispersão, que é uma fórmula integral relacionando um processo dispersivo a um processo de absorçãa ${ }^{7}$, freqüentemente chamada na literatura de relação de Kramers-Krönig. Tal nomenclatura deve-se aos trabalhos pioneiros de H. A. Kramers e R. de L. Krönig ${ }^{7}$, que obtiveram relações de dispersão para a constante dielétrica e para o índice de refração, cuja aplicação original era na dispersão de raios-X e baseia-se no princípio da Causalidade.

Sob a ação de um estímulo externo, um sistema responde com seu modo característico. A relação entre a resposta e o estímulo é dada por uma função de resposta. Na condição de causalidade ( a função de resposta não pode começar antes da função de estímulo), o teorema de Cauchy é aplicado à função de resposta, $G(\omega)$, gerando a transformada de Hilbert $^{7}$, a partir da qual podem-se derivar as equações:

$$
\begin{aligned}
& \operatorname{Re}[G(\omega)]=\operatorname{Re}[G(\infty)]+\frac{2}{\pi} \mathrm{p} \int_{0}^{\infty} \frac{\omega \operatorname{Im}\left[G\left(\omega^{\prime}\right)\right]}{\omega^{2}-\omega^{1}} \mathrm{~d} \omega^{\prime} \\
& \operatorname{Im}\left[G\left(\omega^{\prime}\right)\right]=-\frac{2 \omega}{\pi} \mathrm{p} \int_{0}^{\infty} \frac{\operatorname{Re}\left[\mathrm{G}\left(\omega^{\prime}\right)\right]}{\omega^{2}-\omega^{1}} \mathrm{~d} \omega^{\prime}
\end{aligned}
$$

onde Re e Im indicam as partes real e imaginária, respectivamente, $\omega$ é uma freqüência angular e p representa o valor principal de Cauchy, ou seja, a integral é calculada excluindo-se o ponto de divergência. 
Essas equações indicam que as partes real e imaginária de uma função de resposta não são independentes quando a função é causal. Foi provado que a polarizabilidade, a função dielétrica complexa, o índice de refração complexo, a refletividade complexa, etc são todos causais. Como resultado, quando um de seus componentes (a parte real ou imaginária) é conhecido, o outro pode ser calculado. É importante notar que na análise de KramersKrönig (KKA) a partir da parte imaginária $(\operatorname{Im}[\mathrm{G}(\omega)])$ para a parte real $(\operatorname{Re}[\mathrm{G}(\omega)])$ é necessário o conhecimento de $\operatorname{Re}[\mathrm{G}(\infty)]$ (por exemplo, $\mathrm{n}_{\infty}$ para o índice de refração complexo).

No presente trabalho, utilizamos a análise de KramersKrönig na obtenção de constantes ópticas a partir de medidas de reflectância especular. Esse tipo de KKA é obtida aplicando-se a equação 13 à equação 5 na sua forma logarítmica (equação 14) resultando a equação 15 onde $\tilde{v}$ é o número de onda:

$$
\text { In } \tilde{\mathrm{r}}=\operatorname{In} \sqrt{\mathrm{R}}+\mathrm{i} \delta
$$

$$
\delta\left(\tilde{\mathrm{v}}^{\prime}\right)=-\frac{\tilde{\mathrm{v}}^{\prime}}{\pi} \int_{0}^{\infty} \frac{\operatorname{InR}\left(\tilde{\mathrm{v}}^{\prime}\right)}{\tilde{\mathrm{v}}^{\prime}-\tilde{\mathrm{v}}^{\prime 2}} \mathrm{~d} \tilde{\mathrm{v}}
$$

Existem várias maneiras de se executar a KKA matematicamente. A maneira mais corrente é a chamada KKA subtrativa, apresentada na referência 14. As sub-rotinas da transformada de Kramers-Krönig subtrativa utilizadas no cálculo das constantes ópticas dos materiais apresentados nesse trabalho foram desenvolvidos em nosso laboratório ${ }^{12}$ em ambiente MatLab ${ }^{\circledR}$ com base nos trabalhos bem sucedidos de vários pesquisadores ${ }^{7,13-16}$.

\section{APLICAÇÕES}

\section{Materiais vítreos e cerâmicos}

A espectroscopia de infravermelho (IV) tem sido amplamente utilizada no estudo da estrutura de materiais vítreos como v- $\mathrm{SiO}_{2}, \mathrm{v}-\mathrm{GeO}_{2}$ e v-BeF 2 . Tais materiais foram objeto de um grande número de investigações tanto experimentais quanto teóricas ${ }^{17-21}$. Mais recentemente, além desses vidros típicos, outros tipos de materiais vítreos vêm sendo estudados por espectroscopia no IV, tais quais boratos ${ }^{16}$, fluoretos ${ }^{22}$, fosfatos ${ }^{23}$ e óxidos de metais pesados ${ }^{24}$. A principal vantagem dessa técnica é ser direta na investigação de estruturas. Entretanto, uma vez que não há uma única teoria que permita o cálculo direto da estrutura vítrea a partir das informações espectrais, os modelos estruturais são inferidos e construídos dentro dos limites delineados pelos espectros disponíveis ${ }^{17}$.

Os espectros no IV podem ser obtidos tanto no modo absorção quanto no modo reflexão, porém, uma vez que não é fácil medir espectros de transmissão de materiais que apresentam absorções intensas, a técnica de reflectância especular é mais apropriada e frequentemente aplicada. Como a dispersão do índice de refração tem papel importante numa medida de reflexão, o espectro obtido é completamente diferente daquele obtido através de um experimento de transmissão, que, por sua vez, é fortemente influenciado pelo índice de absorção. Contudo, conforme discutido, o índice de refração complexo e consequentemente a constante dielétrica complexa podem ser determinados a partir do espectro de reflectância através da análise de Kramers-Krönig (KKA) (equações 7, 8, 10, 11 e 15). Isso permite que o espectro de reflectância seja convertido num espectro do índice de absorção, que, por ser diretamente proporcional à absortividade, possibilita a interpretação espectral.

Devido a essa vantagem, a técnica de reflexão especular vêm sendo crescentemente utilizada entre os espectroscopistas. Vários exemplos podem ser citados: (i) Recentemente, Kamitsos ${ }^{16}$ e seus colaboradores demonstraram a potencialidade da técnica para a avaliação quantitativa da estrutura vítrea através de resultados de estudos sobre vidros de boratos alcalinos e de germanatos. (ii) Cariati ${ }^{23}$ e colaboradores também utilizaram a técnica com sucesso para caracterizar a matriz de fosfato em vidros de fosfato de zinco e cobre. (iii) Almeida e seus colaboradores ${ }^{17,23-26}$ fazem amplo uso da espectroscopia especular no estudo de vários tipos de materiais vítreos, desde sílica vítrea a óxidos de metais pesados como chumbo e bismuto.

Como exemplo da utilização da análise de Kramers-Krönig, apresentamos na Figura 2, resultados para a sílica vítrea, obtidos em nosso laboratório.
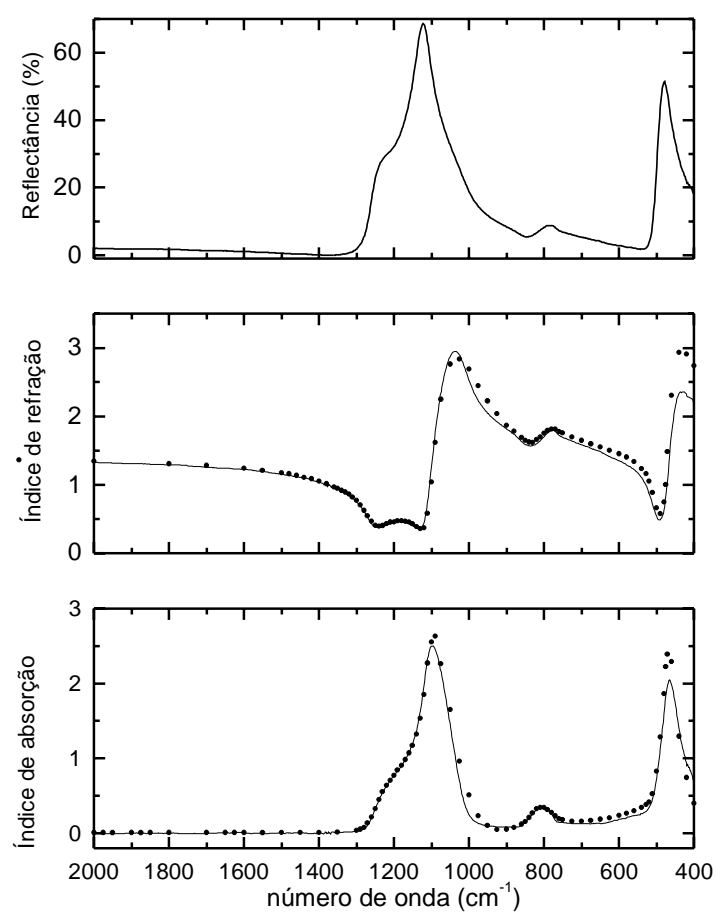

Figura 2. Resultados da análise de Kramers-Krönig para a sílica vítrea: (a) o espectro de reflectância especular; $(b)$ e (c) os índices de refração e de absorção, respectivemente ( (-) extraídos e (.) obtidos na referência 27)

Uma vez que os materiais cerâmicos são duros e apresentam dispersão intensa no infravermelho, a preparação de filmes finos para a obtenção de espectros de transmissão também é bastante complicada. Por outro lado, a obtenção de espectros de reflectância no infravermelho desses materiais é fácil. Cerâmicas são aglomerados de microcristalitos inorgânicos de modo que podem ser tratados como orientados aleatoriamente e isotrópicos. A desvantagem desse método é que ele requer uma superfície lisa da amostra, o que, muitas vezes não é o caso de materiais cerâmicos. Um exemplo de tal procedimento e uma tentativa de interpretação espectral de um material heterogêneo foram dados por Hopfe e seus colaboradores ${ }^{4}$. Eles estudaram a reflexão de cerâmicas comerciais (principalmente a $\alpha-\mathrm{Al}_{2} \mathrm{O}_{3}$ ) com uma superfície macroscopicamente lisa, porém microscopicamente rugosa. Não há muitas alternativas para a caracterização de tal material por infravermelho porque sua forte absortividade impede o uso da técnica convencional de transmissão conforme discutido. Por outro lado, a técnica de ATR apresenta o inconveniente do insuficiente contato óptico entre a amostra de superfície rugosa e o cristal de ATR. Hopfe e seus colaboradores ${ }^{4}$ atingiram seu objetivo através de um modelo óptico de meio efetivo (ar/cerâmica) construído a partir de informações morfológicas. Informações mais detalhadas sobre modelos ópticos de meio efetivo podem ser encontradas nos trabalhos de Grosse $e^{28-30}$ e nas referências por ele citadas. 


\section{Amostras pulverizadas}

Matrizes transparentes, como pastilhas de $\mathrm{KBr}$, e suspensões em Nujol são frequentemente utilizadas em medidas espectrais no infravermelho para materiais pulverizados. Contudo, a técnica da pastilha de $\mathrm{KBr}$ não deve ser usada para ácidos, bases ou sais devido à possibilidade de troca iônica. Dispersões em Nujol, por sua vez, são fortemente contra-indicadas quando suas bandas de absorção encobrem bandas importantes da amostra. Assim, a análise de Kramers-Krönig pode ser um método simples e alternativo. Apresentamos, na Figura 3, resultados obtidos em nosso laboratório para uma pastilha de ácido benzóico. Embora quantidades absolutas não possam ser obtidas devido ao fato da densidade da pastilha ser diferente da densidade real, o espectro do índice de absorção para o ácido benzóico é suficientemente similar ao respectivo espectro de transmitância de modo a permitir a identificação do material.
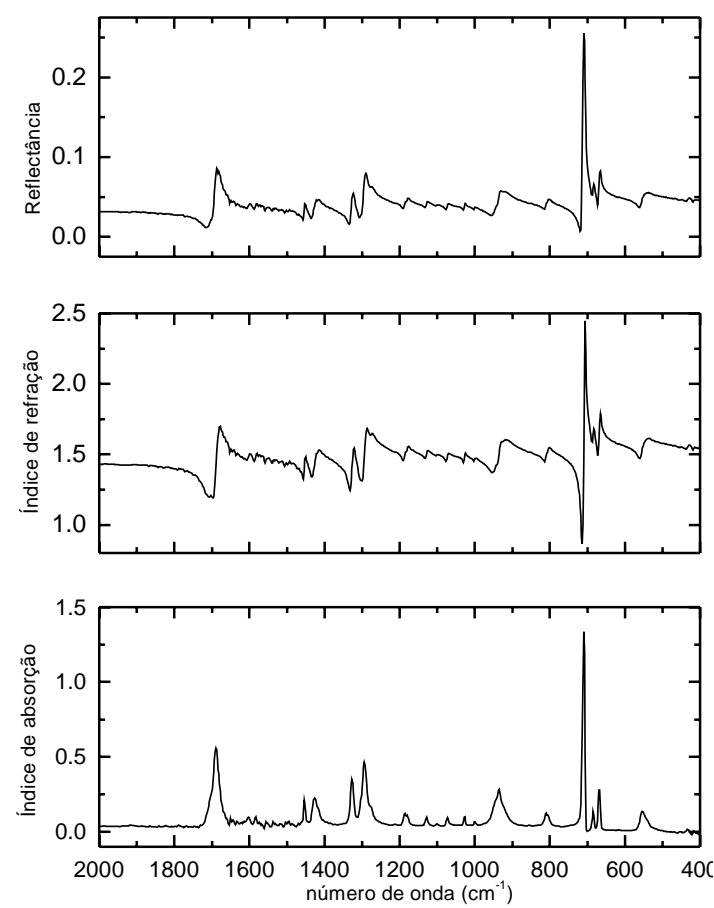

Figura 3. Resultados da análise de Kramers-Krönig para uma pastilha de ácido benzóico: (a) o espectro de reflectância especular; (b) $e(c)$ os índices de refração e de absorção extraídos

Um outro exemplo da aplicação da análise de KramersKrönig a espectros de reflectância de pastilhas de pós foi dado por Karakassides ${ }^{31}$ e seus colaboradores. Eles estudaram a influência dos cátions $\mathrm{Li}^{+}$e $\mathrm{Cs}^{+}$nos modos vibracionais da matriz de $\mathrm{SiO}_{2}$ em montmorillonitas tratadas termicamente.

A determinação de constantes ópticas de pós para fins mais sofisticados que uma simples identificação vem sendo investigada por vários pesquisadores ${ }^{32-34}$. Tal objetivo é bastante válido uma vez que a maioria dos compostos químicos sólidos são policristalinos quando sintetizados ou encontrados na natureza. Analogamente ao caso de cerâmicas rugosas, tais investigações necessariamente passam por modelos ópticos de meio efetivo.

\section{Líquidos}

Existe um grande esforço entre a comunidade científica de fazer com que a espectroscopia de infravermelho se torne uma técnica quantitativa. A maioria dos químicos, mesmo hoje em dia, só presta atenção ao eixo do número de onda quando analisa os seus espectros de infravermelho. O trabalho iniciado por Jones, Bertie e sua equipe é um bom exemplo da tentativa de reversão dessa situação. Trata-se de uma comissão vinculada à "International Union of Pure and Applied Chemistry" que trabalha na busca de padrões secundários de intensidade na região do infravermelho ${ }^{35-44}$. Essa busca se dá necessariamente através da obtenção das constantes ópticas fundamentais de vários líquidos, tais quais: benzeno ${ }^{35}$, tolueno ${ }^{36}$, metanol ${ }^{37}$, clorobenzeno ${ }^{38}$, bromobenzeno ${ }^{39}$, entre outros. Dentre os métodos disponíveis, a análise de Kramers-Krönig é uma das mais estudadas e aplicadas.

A água também tem recebido uma certa atenção nessa linha de pesquisa devido à sua importância fundamental. Tal importância é ilustrada pelo fato de que muito do interesse em suas constantes ópticas no infravermelho tem sido estimulado pela necessidade de cálculos precisos de balanço de massa de energia radiativa através da atmosfera ${ }^{40}$. Além disso, estudos vibracionais de soluções aquosas e de camadas insolúveis na interface ar/água requerem constantes ópticas da água em cálculos de possíveis modelos. Bertie e $\operatorname{Lan}^{39}$ apresentaram as constantes ópticas da água a $25^{\circ} \mathrm{C}$ numa ampla faixa do infravermelho, obtidas a partir de medidas de transmissão e de ATR. Na Figura 4, apresentamos as constantes ópticas da água obtidas em nosso laboratório a partir de dados de reflectância especular via análisa de Kramers-Krönig. Nossos resultados são satisfatórios quando confrontados com os dados de Bertie e Lan ${ }^{40}$. As discrepâncias entre nossos resultados podem estar relacionadas com o fato de suas medidas terem sido feitas a $25^{\circ} \mathrm{C}$ e a nossa em temperatura próxima a $20^{\circ} \mathrm{C}$. Esta hipótese tem sentido uma vez que a maior discrepância entre os espectros do índice $\mathrm{k}$ é observada na posição do pico em baixo número de onda $\left(\sim 600 \mathrm{~cm}^{-1}\right)$ : há um deslocamento de aproximadamente $40 \mathrm{~cm}^{-1}$ entre os espectros.
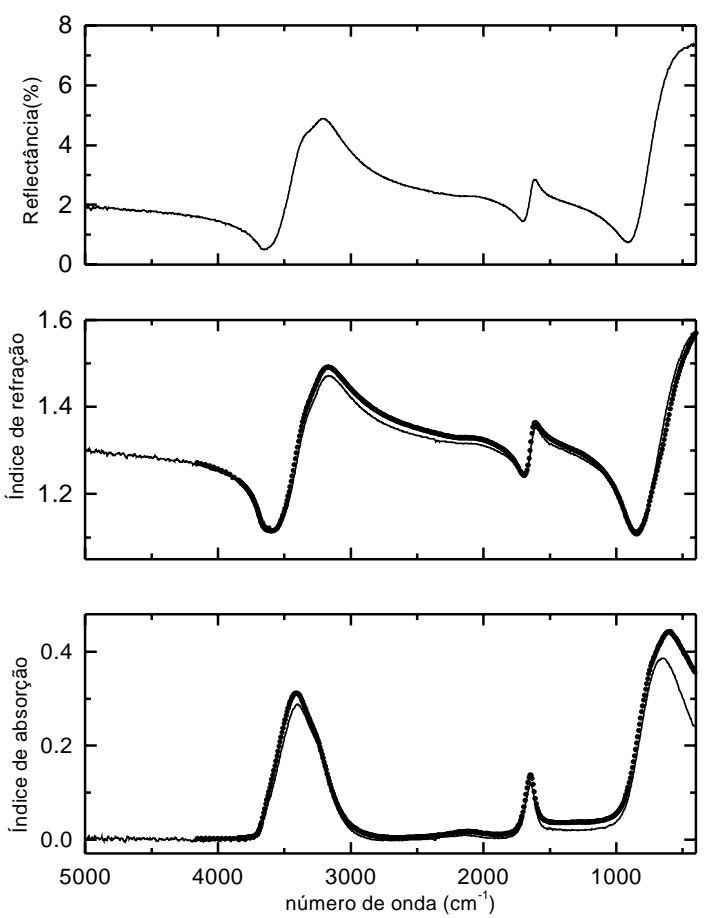

Figura 4: Resultados da análise de Kramers-Krönig para a água: (a) o espectro de reflectância especular; $(b)$ e (c) os indices de refração e de absorção, respectivamente ( ( -) extraídos e (.) obtidos na referência 40)

A obtenção das constantes ópticas de vários outros líquidos também é bastante interessante para a construção de modelos para a interpretação de espectros obtidos por outras técnicas, como, por exemplo, reflexão-absorção. Yamamoto e Ishida ${ }^{7}$ 
exemplificam esse método no seu estudo da orientação e adsorção de perfluorpoliéter em superfícies metálicas. Tal líquido é amplamente utilizado como lubrificante em tecnologias de ponta como computação e aeronaves. Apesar de ser um material orgânico, ele apresenta dispersões relativamente intensas devido às ligações $\mathrm{C}-\mathrm{F}$ e as referentes à função éter na região de $1350-1000 \mathrm{~cm}^{-1}$. No estudo acima, mostrou-se que a função de óptica longitudinal (que será discutida na segunda parte desse artigo) calculada a partir das constantes ópticas do líquido é importante para a interpretação dos espectros de reflexão-absorção.

\section{CONCLUSÃO}

Devido aos avanços tecnológicos na área de informática, a transformada de Kramers-Krönig nunca teve sua aplicação tão palpável quanto ultimamente. Conforme discutimos, ela é uma ferramenta bastante relevante em análises espectrais de materiais cujo espectro de transmissão é de difícil obtenção ou pode ser comprometido pelas técnicas convencionais. Além disso, ela permite a obtenção das constantes ópticas intrínsecas de materiais, que são a base de muitos modelos. É importante salientar que o ferramental computacional desenvolvido em nosso laboratório em ambiente MatLab está a disposição dos interessados mediante solicitação.

\section{AGRADECIMENTOS}

Agradecemos à CAPES e à FAPESP (Processo: 98/11743-2) pelo apoio financeiro e aos Drs. Delson Torikai e Carlos Kennichi Suzuki do Laboratório de Quartzo da Faculdade de Engenharia Mecânica da Unicamp pela amostra de sílica vítrea.

\section{REFERÊNCIAS}

1. Grosse, P.; Offermann, V.; Vib. Spectrosc. 1995, 8, 121

2. Offermann, V.; Grosse, P.; Feuerbacher, M.; Dittmar, G.; Vib. Spectrosc. 1995, 8, 135

3. Culler, S. R.; McKenzie, M. T.; Fina, L. J.; Ishida, H.; Koenig, J. L.; Appl. Spectrosc. 1984, 38, 791

4. Hopfe, V.; Korfe, E. H.; Klobes, P.; Grählert, W.; Appl. Spectrosc. 1993, 47, 423

5. Claybourn, M.; Colomel, P.; Chalmers, J.; Appl. Spectrosc. 1991, 45, 279

6. Abdullah, A. H.; Sherman, W. F.; Vib. Spectrosc. 1997, 13,133

7. Yamamoto, K.; Ishida, H.; Vib. Spectrosc. 1994, 8, 1

8. Meeten, G. H.; Refraction and Extinction of Polymers, In Optical Properties of Polymers, Meeten, G. H., Ed., Elsevier Applied Science Publishers, Essex, 1986, cap. 1

9. Wendlandt, W. W.; Hecht, H. G.; Reflectance Spectroscopy, Interscience Publishers, New York, 1966, cap. 1.

10. Born, M.; Wolf, E.; Principles of Optics, Sixth (Corrected) Edition, Cambridge University Press, Cambridge, 1980, p. 260.
11. Bertie, J. E.; Zhang, S. L.; Keefe, C. D.; Vib. Spectrosc. 1995, 8, 215

12. Trasferetti, B. C.; Dissertação de Mestrado, IQ/UNICAMP, 1998

13. Harbecke, B.; Appl. Phys. A 1986, 40, 151

14. Yamamoto, K.; Masui, A.; Appl. Spectrosc. 1995, 49, 639

15. Ohta, K.; Ishida, H.; Appl. Spectrosc. 1988, 42, 952

16. Kamitsos, E. I.; Yiannopoulos, Y. D.; Varsanis, C. P.; Jain, H.; J. Non-Cryst. Solids 1997, 222, 59

17. Almeida, R. M.; J. Non-Cryst. Solids 1988, 106, 347

18. Payne, M. C.; Inkson, J. C.; J. Non-Cryst. Solids 1984, 68,351

19. Trasferetti, B. C.; Davanzo, C. U.; Appl. Spectrosc. 2000, 54,502

20. de Leeuw, S. W.; Thorpe, M. F.; Phys. Rev. Lett. 1985, 55,2879

21. Sarnthein, J.; Pasquarello, A.; Car, R.; Science 1997, 275, 1925

22. Almeida, R.M.; J. Non-Cryst. Solids 1992, 140, 92

23. Bruni, S.; Cariati, F.; Narducci, D.; Vib. Spectrosc. 1994, 7, 169

24. Kharlamov, A. A.; Almeida, R. M.; Heo, J.; J. Non-Cryst. Solids 1996, 202, 233

25. Almeida, R. M.; Guiton, T. A.; Pantano, C. G.; J. NonCryst. Solids 1990, 119, 238

26. Almeida, R. M.;Phys. Rev. B 1992, 45, 161

27. Philipp, H. R.; J. Appl. Phys. 1979, 50, 1053

28. Sturm, J.; Grosse, P.; Theiss, W.; Z. Phys. B 1991, 83, 361

29. Eickhoff, T.; Grosse, P.; Henkel, S.; Theiss, W.; Z. Phys. B 1992, 88, 17

30. Gorges, E.; Grosse, P.; Theiss, W.; Z. Phys. B 1995, 97, 49

31. Karakassides, M. A.; Petridis, D.; Gournis, D.; Clays and Clay Minerals 1997, 45, 649

32. Volz, F. E.; Appl. Opt. 1983, 22, 1842

33. Lutz, H. D., Müller, B.; Steiner, H. J.; J. Solid State Chem. 1991, 90, 54

34. Pecherromán, C.; Iglesias, J. E.; J. Phys.: Condens. Matter 1994, 6, 7125

35. Bertie, J. E.; Jones, R. N.; Keefe, C. D.; Appl. Spectrosc. 1993, 47, 891

36. Bertie, J. E.; Jones, R. N.; Apelblat, Y.; Appl. Spectrosc. 1994, 48, 127

37. Bertie, J. E.; Zhang, S. L.; Eysel, H. H.; Baluja, S.; Ahmed, M. K.; Appl. Spectrosc. 1993, 47, 1100

38. Bertie, J. E.; Jones, R. N.; Apelblat, Y.; Appl. Spectrosc. 1994, 48, 144

39. Keefe, C. D.; Pittman, J.; Appl. Spectrosc. 1998, 52, 1062

40. Bertie, J. E.; Lan, Z.; Appl. Spectrosc. 1996, 50, 1047

41. Bertie, J. E.; Keefe, C. D.; Jones, R. N.; Can. J. Chem. 1991, 69, 1609

42- Bertie, J. E.; Keefe, C. D.; Jones, R. N.; Mantsch, H. H.; Appl. Spectrosc. 1991, 45, 1233

43. Bertie, J. E.; Keefe, C. D.; Fresen. J. Anal. Chem. 1998, 362, 91

44. Bertie, J. E.; Zhang, S. L.; J. Mol. Struct. 1997, 413, 333. 